\title{
Maintenance of Profile Matchings in Knowledge Bases $^{\star}$
}

\author{
Jorge Martinez Gil ${ }^{1}$, Lorena Paoletti ${ }^{1}$, Gábor Rácz ${ }^{2}$, Attila $\mathrm{Sali}^{2}$, Klaus-Dieter \\ Schewe ${ }^{1}$ \\ 1 Software Competence Center Hagenberg, Softwarepark 21, 4232 Hagenberg, Austria \\ jorge.martinez-gil|lorena.paoletti|kd.schewe@scch.at \\ 2 Alfréd Rényi Institute of Mathematics, P.O.B.127, 1364 Budapest, Hungary \\ gabee33@gmail.com, sali@renyi.hu
}

\begin{abstract}
A profile describes a set of properties, e.g. a set of skills a person may have or a set of skills required for a particular job. Profile matching aims to determine how well a given profile fits to a requested profile. Profiles can be defined by filters in a lattice of concepts derived from a knowledge base that is grounded in description logic, and matching can be realised by assigning values in $[0,1]$ to pairs of such filters: the higher the matching value the better is the fit. In this paper the problem is investigated, whether given a set of filters together with matching values determined by some human expert a matching measure can be determined such that the computed matching values preserve the rankings given by the expert. In the paper plausibility constraints for the values given by an expert are formulated. If these plausibility constraints are satisfied, the problem of determining a ranking-preserving matching measure can be solved.
\end{abstract}

\section{Introduction}

A profile describes a set of properties, and profile matching is concerned with the problem to determine how well a given profile fits to a requested one. Profile matching appears in many application areas such as matching applicants for jobs to job requirements, matching system configurations to requirements specifications, etc.

Taking the profile just as a set of unrelated items is usually not appropriate for the problem, even though many distance measures between sets such as Jaccard or Sørensen-Dice [8] have proven to be useful in ecological applications. The reason is that many dependencies between the properties have to be

\footnotetext{
* The research reported in this paper was supported by the Austrian Forschungsförderungsgesellschaft (FFG) for the Bridge project "Accurate and Efficient Profile Matching in Knowledge Bases" (ACEPROM) under contract [FFG: 841284]. The research reported in this paper has further been supported by the Austrian Ministry for Transport, Innovation and Technology, the Federal Ministry of Science, Research and Economy, and the Province of Upper Austria in the frame of the COMET center SCCH [FFG: 844597].
} 
taken into account. Therefore, in the human resources application area many taxonomies for skills, competences and education such as DISCO [2], ISCED [6] and ISCO [7] have been set up. On the grounds of these application-oriented dictionaries for profile matching a lattice structure for the individual properties can be assumed. This has been exploited by Popov and Jebelean in [13] defining a different asymmetric matching measure on the basis of filters in such lattices.

However, it can well be argued that the hierarchical dependencies in lattices are still insufficient for capturing the exact meaning of the properties in a profile. For instance, it is not common to request just "programming in Java" as a required skill, but it is more likely that further attributes are given such as years of experience associated with the skill, level of complexity of problems addressed with the skill, etc. Therefore, it appears favourable to not only assume a lattice structure, but to exploit sophisticated knowledge representation features for semantic matching problems as advocated by Falk, Mochol and others [3,10]. In our research we adopt this basic assumption how to represent knowledge about properties. That is, we exploit description logics [1] as the basis for knowledge representation using a rather expressive language similar to $\operatorname{SROIQ}(\mathcal{D})[11]$. On these grounds matching top- $k$ queries have been addressed in [12]. In order to generalise matching to knowledge bases we exploit "blowing-up" roles, which means to enrich the concept lattice by inverse images defined by the roles [11]. In Section 2 we briefly review our general approach to profile matching in knowledge bases, formally defining a knowledge representation language and matching measures based on filters. Probabilistic extensions to the theory of matching measures have been investigated in [14].

The second question, which is the core problem handled in this paper concerns the relationship of rankings obtained through the matching measures and the judgements of human experts. An initial idea based on formal concept analysis [4] was already presented in [9] aiming to enrich the knowledge base by additional concepts that would justify the judgement of the human expert. In this paper we investigate the learning of the matching measure. Starting from the set of filters together with matching values or simply rankings determined by some human expert we derive plausibility constraints that should be satisfied to exclude unjustified bias, which could be grounded in the valuation of facts not represented in a knowledge base. Our key result is a proof that the satisfaction of the plausibility constraints implies the existence of a matching measure that preserves the human rankings. This key contribution will be presented in Section 3 . We conclude with a brief summary.

\section{Profile Matching in Knowledge Bases}

In this section we present the formal definitions underlying our approach to profile matching in knowledge bases. We will start with the general approach to knowledge representation, proceed with the representation of profiles, and discuss filter-based matching. 


\subsection{Knowledge Representation}

For the representation of knowledge we adopt the fundamental distinction between terminological and assertional knowledge that has been used in description logics since decades. For the former one we define a language, which defines the TBox of a knowledge base, while the instances define a corresponding ABox.

A TBox consists of concepts and roles. In addition, we will permit the denotation of individuals as supported by $\operatorname{SROIQ}(\mathcal{D})$ [1] and OWL2 [5]. For this assume that $C_{0}, I_{0}$ and $R_{0}$ represent not further specified sets of basic concepts, individuals and roles, respectively. Then atomic concepts $A$, concepts $C$ and roles $R$ are defined by the following grammar:

$$
\begin{aligned}
& R=R_{0}\left|R_{0}^{-}\right| R_{1} \circ R_{2} \\
& \left.A=C_{0}|\top| \geq m . R \text { (with } m>0\right) \mid\left\{I_{0}\right\} \\
& C=A|\neg C| C_{1} \sqcap C_{2}\left|C_{1} \sqcup C_{2}\right| \exists R . C \mid \forall R . C
\end{aligned}
$$

Definition 1. A TBox is a finite set $\mathcal{T}$ of assertions of the form $C_{1} \sqsubseteq C_{2}$ with concepts $C_{1}$ and $C_{2}$ as defined by the grammar above.

Each assertion $C_{1} \sqsubseteq C_{2}$ in a TBox $\mathcal{T}$ is called a subsumption axiom. Note that Definition 1 only permits subsumption between concepts, not between roles, though it is possible to define more complex terminologies that also permit role subsumption. As usual, we use several shortcuts: (1) $C_{1} \equiv C_{2}$ can be used instead of $C_{1} \sqsubseteq C_{2} \sqsubseteq C_{1},(2) \perp$ is a shortcut for $\neg \top,(3)\left\{a_{1}, \ldots, a_{n}\right\}$ is a shortcut for $\left\{a_{1}\right\} \sqcup \cdots \sqcup\left\{a_{n}\right\},(4) \leq m . R$ is a shortcut for $\neg \geq m+1 . R$, and $(5)=m . R$ is a shortcut for $\geq m \cdot R \sqcap \leq m \cdot R$.

Definition 2. A structure $\mathcal{S}$ for a TBox $\mathcal{T}$ consists of a non-empty set $\mathcal{O}$ together with subsets $\mathcal{S}\left(C_{0}\right) \subseteq \mathcal{O}$ and $\mathcal{S}\left(R_{0}\right) \subseteq \mathcal{O} \times \mathcal{O}$ for all basic concepts $R_{0}$ and basic roles $R_{0}$, respectively, and individuals $\bar{a} \in \mathcal{O}$ for all $a \in I_{0}$. $\mathcal{O}$ is called the base set of the structure.

We first extend the interpretation of basic concepts and roles and to all concepts and roles as defined by the grammar above, i.e. for each concept $C$ we define a subset $\mathcal{S}(C) \subseteq \mathcal{O}$, and for each role $R$ we define a subset $\mathcal{S}(R) \subseteq \mathcal{O} \times \mathcal{O}$ as follows:

$$
\begin{gathered}
\mathcal{S}\left(R_{0}^{-}\right)=\left\{(y, x) \mid(x, y) \in \mathcal{S}\left(R_{0}\right)\right\} \\
\mathcal{S}\left(R_{1} \circ R_{2}\right)=\left\{(x, z) \mid \exists y .(x, y) \in \mathcal{S}\left(R_{1}\right) \wedge(y, z) \in \mathcal{S}\left(R_{2}\right)\right. \\
\mathcal{S}(\top)=\mathcal{O} \quad \mathcal{S}(\{a\})=\{\bar{a}\} \quad \mathcal{S}(\neg C)=\mathcal{O}-\mathcal{S}(C) \\
\mathcal{S}(\geq m . R)=\{x \in \mathcal{O} \mid \#\{y \mid(x, y) \in \mathcal{S}(R)\} \geq m\} \\
\mathcal{S}\left(C_{1} \sqcap C_{2}\right)=\mathcal{S}\left(C_{1}\right) \cap \mathcal{S}\left(C_{2}\right) \quad \mathcal{S}\left(C_{1} \sqcup C_{2}\right)=\mathcal{S}\left(C_{1}\right) \cup \mathcal{S}\left(C_{2}\right) \\
\mathcal{S}(\exists R . C)=\{x \in \mathcal{O} \mid(x, y) \in \mathcal{S}(R) \text { for some } y \in \mathcal{S}(C)\} \\
\mathcal{S}(\forall R . C)=\{x \in \mathcal{O} \mid(x, y) \in \mathcal{S}(R) \Rightarrow y \in \mathcal{S}(C) \text { for all } y\}
\end{gathered}
$$

Definition 3. An $A B o x$ for a TBox $\mathcal{T}$ is a finite structure $\mathcal{S}$, such that $\mathcal{S}\left(C_{1}\right) \subseteq$ $\mathcal{S}\left(C_{2}\right)$ holds for all assertions $C_{1} \sqsubseteq C_{2}$ in $\mathcal{T}$. 
For the following we always consider a concept $C$ in a TBox as representation of abstract properties, e.g. "knowledge of Java", and individuals in the ABox as concrete properties such as the "Java knowledge of Lara". Therefore, given an ABox a profile is simply a subset of the base set $\mathcal{O}$.

\section{$2.2 \quad$ Filter-Based Matching}

Obviously, the concepts in a TBox define a lattice with $\sqcap$ and $\sqcup$ as operators for meet and join, and $\sqsubseteq$ for the partial order. So let us abstract for a moment from the specific definition of the knowledge base by TBox and ABox and assume to be given a lattice $(\mathcal{L}, \leq)$.

Definition 4. A filter in a lattice $(\mathcal{L}, \leq)$ is a non-empty subset $\mathcal{F} \subseteq \mathcal{L}$, such that for all $C, C^{\prime}$ with $C \leq C^{\prime}$ whenever $C \in \mathcal{F}$ holds, then also $C^{\prime} \in \mathcal{F}$ holds.

If $P \subseteq \mathcal{O}$ is a profile, then $P$ defines in a natural way a filter $\mathcal{F}$ of the lattice $\mathcal{L}$ of concepts: $\mathcal{F}=\{C \in \mathcal{L} \mid \exists p \in P . p \in \mathcal{S}(C)\}$. Therefore, for determining matching relations we can concentrate on filters $\mathcal{F}$ in a lattice.

Definition 5. Let $(\mathcal{L}, \leq)$ be a lattice, and let $\mathbb{F} \subseteq \mathcal{P}(\mathcal{L})$ denote the set of filters in this lattice.

A relative weight measure on $\mathcal{L}$ is a function $m: \mathcal{P}(\mathcal{L}) \rightarrow[0,1]$ satisfying (1) $m(\mathcal{L})=1$, and $(2) m\left(\bigcup_{i \in I} A_{i}\right)=\sum_{i \in I} m\left(A_{i}\right)$ for pairwise disjoint $A_{i}(i \in I)$.

A matching measure is a function $\mu: \mathbb{F} \times \mathbb{F} \rightarrow[0,1]$ such that $\mu\left(\mathcal{F}_{1}, \mathcal{F}_{2}\right)=$ $m\left(\mathcal{F}_{1} \cap \mathcal{F}_{2}\right) / m\left(\mathcal{F}_{2}\right)$ holds for some relative weight measure $m$ on $\mathcal{L}$.

Example 1. The matching measure $\mu_{p j}$ defined in [13] uses simply cardinalities: $\mu_{p j}\left(\mathcal{F}_{1}, \mathcal{F}_{2}\right)=\#\left(\mathcal{F}_{1} \cap \mathcal{F}_{2}\right) / \# \mathcal{F}_{2}$. Thus, it is defined by the relative weight measure $m$ on $\mathcal{L}$ with $m(A)=\# A / \# \mathcal{L}$.

It is easy to see that every matching measure $\mu$ is defined by weights $w(C)=$ $m(\{C\}) \in[0,1]$ for the elements $C \in \mathcal{L}$. With this we immediately obtain $m(\mathcal{F})=\sum_{C \in \mathcal{F}} w(C)$ and thus $\mu\left(\mathcal{F}_{1}, \mathcal{F}_{2}\right)=\sum_{C \in \mathcal{F}_{1} \cap \mathcal{F}_{2}} w(C) \cdot\left(\sum_{C \in \mathcal{F}_{2}} w(C)\right)^{-1}$.

Example 2. Take a simple lattice $\mathcal{L}$ with only five elements: $\mathcal{L}=\left\{C_{1}, C_{2}, C_{3}\right.$, $\left.C_{4}, C_{5}\right\}$ with $C_{5}<C_{2}<C_{1}$ and $C_{5}<C_{4}<C_{3}<C_{1}$. Then we obtain seven filters for this lattice, each generated by one or two elements of the lattice.

If we now define weights $w\left(C_{1}\right)=\frac{1}{10}, w\left(C_{2}\right)=\frac{3}{10}, w\left(C_{3}\right)=\frac{1}{5}, w\left(C_{4}\right)=\frac{3}{10}$, $w\left(C_{5}\right)=\frac{1}{10}$, then we obtain the matching measure values $\mu(\mathcal{F}, \mathcal{G})$ shown in Table 1 . In the table the row label is $\mathcal{F}$ and the column label is $\mathcal{G}$.

The matching measures introduced so far are based solely on filters in a lattice, but a TBox is more than its concept lattice. In order to fully exploit the knowledge represented in a TBox we use blow-up operators. Formally, if $C$ is a concept, for which $C \sqsubseteq \exists R . C^{\prime}$ holds, then for any subconcept $C^{\prime \prime} \sqsubseteq C^{\prime}$ we can define the subconcept $\mathrm{bl}_{R, C^{\prime \prime}}(C)=C \sqcap \exists R . C^{\prime \prime}$ of $C$, which is called the result of the blow-up of $R$ with respect to $C^{\prime \prime}$ on the concept $C$. 


\begin{tabular}{|r||c|c|c|c|c|c|c|}
\hline & $\left\langle C_{1}\right\rangle$ & $\left\langle C_{2}\right\rangle$ & $\left\langle C_{3}\right\rangle$ & $\left\langle C_{2}, C_{3}\right\rangle$ & $\left\langle C_{4}\right\rangle$ & $\left\langle C_{2}, C_{4}\right\rangle$ & $\left\langle C_{5}\right\rangle$ \\
\hline \hline$\left\langle C_{1}\right\rangle$ & 1 & $\frac{1}{4}$ & $\frac{1}{3}$ & $\frac{1}{6}$ & $\frac{1}{6}$ & $\frac{1}{9}$ & $\frac{1}{10}$ \\
\hline$\left\langle C_{2}\right\rangle$ & 1 & 1 & $\frac{1}{3}$ & $\frac{2}{3}$ & $\frac{1}{6}$ & $\frac{4}{9}$ & $\frac{2}{5}$ \\
\hline$\left\langle C_{3}\right\rangle$ & 1 & $\frac{1}{4}$ & 1 & $\frac{1}{2}$ & $\frac{1}{2}$ & $\frac{1}{3}$ & $\frac{3}{10}$ \\
\hline$\left\langle C_{2}, C_{3}\right\rangle$ & 1 & 1 & 1 & 1 & $\frac{1}{2}$ & $\frac{2}{3}$ & $\frac{3}{5}$ \\
\hline$\left\langle C_{4}\right\rangle$ & 1 & $\frac{1}{4}$ & 1 & $\frac{1}{2}$ & 1 & $\frac{2}{3}$ & $\frac{2}{5}$ \\
\hline$\left\langle C_{2}, C_{4}\right\rangle$ & 1 & 1 & 1 & 1 & 1 & 1 & $\frac{9}{10}$ \\
\hline$\left\langle C_{5}\right\rangle$ & 1 & 1 & 1 & 1 & 1 & 1 & 1 \\
\hline
\end{tabular}

Table 1. A matching measure $\mu$ on the lattice $\mathcal{L}$

In particular, this becomes relevant, if $C^{\prime \prime}$ is defined by individuals, say $C^{\prime \prime}=\left\{a_{1}, \ldots, a_{n}\right\}$. Any subsumption between subconcepts of $C^{\prime}$ naturally induces subsumption on these blown-up subconcepts of $C$, i.e. we have $C_{1} \sqsubseteq$ $C_{2} \Rightarrow \mathrm{bl}_{R, C_{1}}(C) \sqsubseteq \mathrm{bl}_{R, C_{2}}(C)$. By means of the blow-up operators we bring the information carried by the roles into additional concepts, to which the matching measures as discussed before can be applied.

\section{Matching Analysis}

Let $\mathcal{L}$ be a lattice with profiles defined by filters. Let $\mathbb{F}$ denote the set of all filters. Note that each filter $\mathcal{F} \in \mathbb{F}$ is uniquely determined by its minimal elements, so we can write $\mathcal{F}=\left\langle C_{1}, \ldots, C_{k}\right\rangle$. The matching knowledge of a human expert can be represented be a partial mapping $h: \mathbb{F} \times \mathbb{F} \rightarrow[0,1]$. Though human experts will hardly ever provide complete information, we will assume in the sequel that $h$ is total.

The general question is whether there exists a matching measure $\mu$ on $\mathbb{F}$ as defined before such that $\mu(\mathcal{F}, \mathcal{G})=h(\mathcal{F}, \mathcal{G})$ holds for all pairs of filters. As the matching values as such are merely used to determine rankings whereas their concrete value is of minor importance, this problem can be weakened to find a ranking-preserving matching measure $\mu$ on $\mathbb{F}$, i.e. the matching measure should imply the same rankings.

Definition 6. A matching measure $\mu$ on $\mathbb{F}$ is called ranking-preserving with respect to $h: \mathbb{F} \times \mathbb{F} \rightarrow[0,1]$ iff

(1) for all filters $\mu\left(\mathcal{F}_{1}, \mathcal{G}\right) \geq \mu\left(\mathcal{F}_{2}, \mathcal{G}\right)$ holds, whenever $h\left(\mathcal{F}_{1}, \mathcal{G}\right) \geq h\left(\mathcal{F}_{2}, \mathcal{G}\right)$ holds, and

(2) for all filters $\mu\left(\mathcal{F}, \mathcal{G}_{1}\right) \geq \mu\left(\mathcal{F}, \mathcal{G}_{2}\right)$ holds, whenever $h\left(\mathcal{F}, \mathcal{G}_{1}\right) \geq h\left(\mathcal{F}, \mathcal{G}_{2}\right)$ holds.

\subsection{Plausibility Constraints}

We are looking for plausibility constraints for the mapping $h$ that should be satisfied in the absence of bias, i.e. the assessment of the human expert is not 
grounded in hidden concepts. If such plausibility conditions are satisfied we explore the existence of a ranking-preserving matching measure $\mu$. First we show the following simple lemma.

Lemma 1. Let $\mu$ be a matching measure on $\mathbb{F}$. Then for all filters $\mathcal{F}, \mathcal{F}_{1}, \mathcal{F}_{2}, \mathcal{G} \in$ $\mathbb{F}$ the following conditions hold:

(1) $\mu(\mathcal{F}, \mathcal{G})=1$ for $\mathcal{G} \subseteq \mathcal{F}$.

(2) $\mu(\mathcal{F}, \mathcal{G}) \leq \mu(\mathcal{F}, \mathcal{G}-\{C\})$ holds for $C \notin \mathcal{F}$.

(3) $\mu(\mathcal{F}, \mathcal{G}) \leq \mu(\mathcal{F} \cup\{C\}, \mathcal{G} \cup\{C\})$.

(4) If $\mu\left(\mathcal{F}_{1}, \mathcal{G}\right)<\mu\left(\mathcal{F}_{2}, \mathcal{G}\right)$ holds, then for every $C \in \mathcal{G}-\mathcal{F}_{1}-\mathcal{F}_{2}$ we have $\mu\left(\mathcal{F}_{1} \cup\{C\}, \mathcal{G}\right)<\mu\left(\mathcal{F}_{2} \cup\{C\}, \mathcal{G}\right)$.

(5) If $\mathcal{F} \cap \mathcal{F}_{1} \cap \mathcal{G}=\mathcal{F} \cap \mathcal{F}_{2} \cap \mathcal{G}$ holds, then $\mu\left(\mathcal{F}_{1}, \mathcal{G}\right)>\mu\left(\mathcal{F}_{2}, \mathcal{G}\right) \Leftrightarrow \mu\left(\mathcal{F}, \mathcal{F}_{1} \cap \mathcal{G}\right)<$ $\mu\left(\mathcal{F}, \mathcal{F}_{2} \cap \mathcal{G}\right)$.

Proof. Properties (1) and (4) are obvious from the definition of matching measures (Definition 5).

For property (2) let $C \in \mathcal{G}$ without loss of generality. Then we have

$$
\mu(\mathcal{F}, \mathcal{G})=\frac{m(\mathcal{F} \cap(\mathcal{G}-\{C\}))}{m(\mathcal{G}-\{C\})+w(C)} \leq \frac{m(\mathcal{F} \cap(\mathcal{G}-\{C\}))}{m(\mathcal{G}-\{C\})}=\mu(\mathcal{F}, \mathcal{G}-\{C\}) .
$$

For property (3) the case $C \notin \mathcal{F}$ is trivial. In case $C \in \mathcal{G}-\mathcal{F}$ holds, we get

$$
\mu(\mathcal{F}, \mathcal{G})=\frac{m(\mathcal{F} \cap \mathcal{G})}{m(\mathcal{G})} \leq \frac{m(\mathcal{F} \cap \mathcal{G})+w(C)}{m(\mathcal{G})}=\mu(\mathcal{F} \cup\{C\}, \mathcal{G} \cup\{C\})
$$

In case $C \notin \mathcal{G}$ first note that for any values $a, b, c$ with $a \leq b$ we get $a b+a c \leq$ $a b+b c$ and thus $\frac{a}{b} \leq \frac{a+c}{b+c}$. Thus, we get

$$
\mu(\mathcal{F}, \mathcal{G})=\frac{m(\mathcal{F} \cap \mathcal{G})}{m(\mathcal{G})} \leq \frac{m(\mathcal{F} \cap \mathcal{G})+w(C)}{m(\mathcal{G})+w(C)}=\mu(\mathcal{F} \cup\{C\}, \mathcal{G} \cup\{C\}) .
$$

For property (5) both sides of the equivalence are equivalent to $m\left(\mathcal{F}_{1} \cap \mathcal{G}\right)>$ $m\left(\mathcal{F}_{2} \cap \mathcal{G}\right)$, which completes the proof.

Informally phrased property (1) states that whenever all requirements in a requested profile $\mathcal{G}$ (maybe even more) are satisfied by a given profile $\mathcal{F}$, then $\mathcal{F}$ is a perfect match for $\mathcal{G}$. Property (2) states that if a requirement not satisfied by a given profile $\mathcal{F}$ is removed from the requested profile $\mathcal{G}$, the given profile will become a better match for the restricted profile. Property (3) covers two cases. If $C \in \mathcal{G}$ holds, then simply the profile $\mathcal{F} \cup\{C\}$ satisfies more requirements than $\mathcal{F}$, so the matching value should increase. The case $C \notin \mathcal{G}$ is a bit more tricky, as the profile $\mathcal{G} \cup\{C\}$ contains an additional requirement, which is satisfied by the enlarged profile $\mathcal{F} \cup\{C\}$. In this case the matching value should increase, because the percentage of requirements that are satisfied increases. Property (4) states that if the given profile $\mathcal{F}_{1}$ is better suited for the required profile $\mathcal{G}$ than 
the given profile $\mathcal{F}_{2}$, then relative to $\mathcal{G}$ the profile $\mathcal{F}_{2}$ is less over-qualified than $\mathcal{F}_{1}$ for any other required profile $\mathcal{F}$, provided the intersections of $\mathcal{F} \cap \mathcal{G}$ with the two given profiles coincide.

Thus, disregarding for the moment our theory of matching measures, all four properties in Lemma 1 appear to be reasonable. Therefore, we require them as plausibility constraints that a human-defined mapping $h: \mathbb{F} \times \mathbb{F} \rightarrow[0,1]$ should satisfy:

(1) $h(\mathcal{F}, \mathcal{G})=1$ for $\mathcal{G} \subseteq \mathcal{F}$,

(2) $h(\mathcal{F}, \mathcal{G}) \leq h(\mathcal{F}, \mathcal{G}-\{C\})$ for any concept $C \notin \mathcal{F}$, and

(3) $h(\mathcal{F}, \mathcal{G}) \leq h(\mathcal{F} \cup\{C\}, \mathcal{G} \cup\{C\})$ for any concept $C$.

(4) If $h\left(\mathcal{F}_{1}, \mathcal{G}\right)<h\left(\mathcal{F}_{2}, \mathcal{G}\right)$ holds, then for every $C \in \mathcal{G}-\mathcal{F}_{1}-\mathcal{F}_{2}$ we have $h\left(\mathcal{F}_{1} \cup\{C\}, \mathcal{G}\right)<h\left(\mathcal{F}_{2} \cup\{C\}, \mathcal{G}\right)$.

(5) If $\mathcal{F} \cap \mathcal{F}_{1} \cap \mathcal{G}=\mathcal{F} \cap \mathcal{F}_{2} \cap \mathcal{G}$ holds, then $h\left(\mathcal{F}_{1}, \mathcal{G}\right)>h\left(\mathcal{F}_{2}, \mathcal{G}\right) \Leftrightarrow h\left(\mathcal{F}, \mathcal{F}_{1} \cap \mathcal{G}\right)<$ $h\left(\mathcal{F}, \mathcal{F}_{2} \cap \mathcal{G}\right)$.

\subsection{Linear Inequations}

Let $h$ be a human-defined matching measure that satisfies the plausibility constraints. Assume the lattice $\mathcal{L}$ contains $n+2$ elements $C_{0}, \ldots, C_{n+1}$ with topand bottom elements $C_{0}$ and $C_{n+1}$, respectively.

Fixing a requested profile $\mathcal{G}$, then $h\left(\mathcal{F}_{1}, \mathcal{G}\right)<h\left(\mathcal{F}_{2}, \mathcal{G}\right)$ defines a linear inequation of the form $\sum_{x \in U} x<\sum_{x \in V} x$ with $U=\left\{w(C) \mid C \in \mathcal{F}_{1} \cap \mathcal{G}\right\}$ and $V=\left\{w(C) \mid C \in \mathcal{F}_{2} \cap \mathcal{G}\right\}$. In these inequalities we may remove summands $w(C)$ on both sides for $C \in \mathcal{F}_{1} \cap \mathcal{F}_{2} \cap \mathcal{G}$. In particular, $w\left(C_{0}\right)$ never appears in the inequalities. Without loss of generality we can also ignore $C_{n+1}$, as it only appears for the trivial case.

If we fix a given profile $\mathcal{F}$, we also obtain linear inequalities of the form $\sum_{x \in U} x<\sum_{x \in V} x$ with disjoint sets $U$ and $V$ corresponding to sets of weights of concepts. If all these inequalities can be satisfied, then clearly the solution defines a matching measure $\mu$ that is order-preserving with respect to $h$.

The "worst case" arises, if we have a linear order on the set of all terms $\sum_{i \in I} x_{i}$, where $x_{i}$ represents $w\left(C_{i}\right)$ and $I \subseteq\{1, \ldots, n\}$. This arises for the lattice $\mathcal{L}$, in which all $C_{i}(i=1, \ldots, n)$ are pairwise incomparable. For all other lattices we can extend the set of inequalities derived from $h$ to the "worst case".

So we reduce the problem of finding a ranking-preserving matching measure to a problem of solving a set of linear inequalities. Thus, let $\mathcal{P}$ be a linear order on the set of terms $\left\{\sum_{i \in I} x_{i} \mid I \subseteq\{1, \ldots, n\}\right\}$. We say that $\mathcal{P}$ is realisable iff there is a substitution $v:\left\{x_{1}, \ldots, x_{n}\right\} \rightarrow \mathbb{R}^{+}$of the variables by positive real numbers such that $\sum_{i \in I} x_{i}$ precedes $\sum_{j \in J} x_{j}$ in $\mathcal{P}$ iff $\sum_{i \in I} v\left(x_{i}\right)<\sum_{j \in J} v\left(x_{j}\right)$ holds. As all sums are finite, it is no loss of generality to seek substitutions by rational numbers, and further using the common denominator it suffices to consider positive integers only.

As $\mathcal{P}$ is defined by $h$ we can assume that $\sum_{i \in I} x_{i}$ precedes $\sum_{j \in J} x_{j}$ for $I \subset J$. We can then also extend $\mathcal{P}$ to a partial order $\hat{\mathcal{P}}$ on multisets of variables 
by adding the same variable(s) to both sides. Clearly, $\mathcal{P}$ is realisable iff $\hat{\mathcal{P}}$ is realisable. For convenience we introduce the notation $U \prec V$ for multisets $U, V$ over $\left\{x_{1}, \ldots, x_{n}\right\}$ to denote the inequality $\sum_{x_{i} \in U} m_{U}\left(x_{i}\right) x_{i}<\sum_{x_{j} \in V} m_{V}\left(x_{j}\right) x_{j}$, where $m_{U}$ and $m_{V}$ are the multiplicities for the two multisets.

Theorem 1. $\mathcal{P}$ is realisable iff there is no positive integer combination of inequalities in $\mathcal{P}$ that results in $A \prec B$ with $B \subseteq A$ as multisets, i.e. $m_{B}\left(x_{i}\right) \leq$ $m_{A}\left(x_{i}\right)$ for all $i=1, \ldots, n$.

Proof (sketch). The necessity of the condition is obvious. For the sufficiency we use Fourier-Motzkin elimination.

\subsection{Derivation of Matching Measures}

We now use Theorem 1 to prove our main result:

Theorem 2. Let h be a human-defined matching measure that satisfies the plausibility constraints. Then there esists a matching measure $\mu$ that is rankingpreserving with respect to $h$.

Proof. Assume that $\mathcal{P}$ is not realisable. Then according to Theorem 1 there exist inequalities $U_{1}<V_{1}, \ldots, U_{k}<V_{k}$ in $\mathcal{P}$ such that $V=\biguplus_{i=1}^{k} V_{i} \subseteq \biguplus_{i=1}^{k} U_{i}=U$ as multisets and

$$
\sum_{x \in U} \sum_{j=i}^{k} m_{U_{j}}(x) x<\sum_{x \in V} \sum_{j=i}^{k} m_{V_{j}}(x) x .
$$

Let this system of inequalities be minimal, so each subset violates the condition in Theorem 1. Taking the inequalities in the some order let

$$
A_{i}=\sum_{x} \sum_{j=1}^{i} m_{U_{j}}(x) x \quad \text { and } \quad B_{i}=\sum_{x} \sum_{j=1}^{i} m_{V_{j}}(x) x .
$$

Then for $i<k$ there always exists some $x$ with $\sum_{j=1}^{i} m_{U_{j}}(x)<\sum_{j=1}^{i} m_{V_{j}}(x)$, while $A_{i} \prec B_{i}$. On the other hand $\sum_{j=1}^{k} m_{U_{j}}(x) \geq \sum_{j=1}^{k} m_{V_{j}}(x)$, while $A_{k} \prec B_{k}$.

Let $V_{i}^{\prime}$ be the multiset $B_{i}-A_{i}$, i.e. the multiset of all $x$ with $m_{B_{i}}(x)>m_{A_{i}}(x)$ such that $m_{V_{i}^{\prime}}(x)=m_{B_{i}}(x)-m_{A_{i}}(x)$ holds. Each $x \in V_{i}^{\prime}$ is a witness for the violation of the condition in Theorem 1 . In particular, we have $V_{i}^{\prime} \neq \emptyset$ for all $i<k$, but $V_{k}^{\prime}=\emptyset$.

Let $V_{i+1}^{\prime \prime}=V_{i} \cap V_{i+1}$ as multisets, so $m_{V_{i+1}^{\prime \prime}}(x)=\min \left(m_{V_{i}^{\prime}}(x), m_{V_{i+1}^{\prime}}(x)\right)$, i.e. $x$ will at most be added to $B_{i}$ to give $B_{i+1}^{i+1}$, but not to $A_{i}$. In particular, $V_{i+1}^{\prime \prime} \subseteq V_{i}^{\prime}$. Take the complement $U_{i+1}^{\prime}$ such that $V_{i}^{\prime}=U_{i+1}^{\prime} \uplus V_{i+1}^{\prime \prime}$.

As $U_{i}<V_{i}$ is in $\mathcal{P}$, we also have $U_{i}^{\prime}<V_{i}$ in $\mathcal{P}$ for all $i>1\left(U_{1}^{\prime}\right.$ is not yet defined). 
Let $B_{1}^{\prime}=V_{1}=V_{1}^{\prime}$. Then proceed inductively defining $W_{i}=B_{i}^{\prime}-U_{i+1}^{\prime}$ as well as $A_{i+1}^{\prime}=U_{i+1}^{\prime} \uplus W_{i}$ and $B_{i+1}^{\prime}=V_{i+1} \uplus W_{i}$, which gives $A_{i+1}^{\prime}<B_{i+1}^{\prime}$ in $\hat{\mathcal{P}}$ and $B_{i}^{\prime}=A_{i+1}^{\prime}$. That is, we obtain a chain

$$
B_{1}^{\prime}=A_{2}^{\prime}<B_{2}^{\prime}=\cdots<B_{k-1}^{\prime}=A_{k}^{\prime}<B_{k}^{\prime} .
$$

Complement these definitions by $U_{1}^{\prime}=B_{k}^{\prime} \cap U_{1}=A_{1}^{\prime}$, and $X_{0}=B_{k}^{\prime}-U_{1}=$ $X_{1}$. Proceed inductively defining

$$
C_{1}=U_{1}^{\prime} \uplus X_{0} \prec V_{1} \uplus X_{1}=B_{1}^{\prime} \uplus X_{1}=D_{1} \text { and } X_{i+1}=X_{i}-\left(U_{i+1}-A_{i+1}^{\prime}\right)
$$

This gives $C_{i+1}=A_{i+1}^{\prime} \uplus X_{i} \prec B_{i+1}^{\prime} \uplus X_{i+1}=D_{i+1}$ and $C_{i+1}=D_{i}$. Finally, due to this construction we also have $X_{i} \supseteq X_{i+1}$ for all $i$ and $X_{k}=\emptyset$. This implies $D_{k}=C_{1}$, which means we have a cycle in $\hat{\mathcal{P}}$ contradicting the fact that it is a partial order. Therefore, $\mathcal{P}$ must be realisable.

Example 3. (1) To illustrate the construction in the proof take the inequalities $x_{1}+x_{2}<x_{3}+x_{4}, x_{2}+x_{3}<x_{5}, x_{4}+x_{5}<x_{1}+x_{3}$ and $x_{3}<x_{2}$ in $\mathcal{P}$ which satisfy the condition in Theorem 1 . From these we construct first the following inequalities in $\hat{\mathcal{P}}: x_{3}+x_{4}<x_{4}+x_{5}, x_{4}+x_{5}<x_{1}+x_{3}$ and $x_{1}+x_{3}<x_{1}+x_{2}$. As the last right hand side is $U_{1}$, we get $X_{0}=\emptyset$, which defines the additional inequality $x_{1}+x_{2}<x_{3}+x_{4}$. These four inequalities define the contradictory cycle in $\hat{\mathcal{P}}$.

(2) If the third inequality had been $x_{4}+x_{5}<x_{1}+x_{2}+x_{3}$ instead, the constructed inequalities in $\hat{\mathcal{P}}$ would have been $x_{3}+x_{4}<x_{4}+x_{5}, x_{4}+x_{5}<$ $x_{1}+x_{2}+x_{3}$ and $x_{1}+x_{2}+x_{3}<x_{1}+2 x_{2}$. This defines $X_{0}=\left\{x_{2}\right\}$, which gives the addition inequality $x_{1}+2 x_{2}<x_{2}+x_{3}+x_{4}$ and the modified inequality $x_{2}+x_{3}+x_{4}<x_{4}+x_{5}$, which again defines a cycle.

Note that our main result only states the existence of a ranking preserving matching measure $\mu$. However, we obtain solutions for the linear inequations defined by $h$ by minimising $x_{1}+\cdots+x_{n}-1$ under the conditions $\sum_{x_{j} \in V} x_{j}-$ $\sum_{x_{i} \in U} x_{i}>0$. For this linear optimisation problem the well-known simplex algorithm can be exploited.

\section{Conclusion}

In this paper we addressed the problem to determine matching measures for profiles that produce rankings, which are in accordance with the measures given by a human expert or at least imply the same rankings. For this we analysed linear inequalities that result from the human-defined rankings. We could show that if certain plausibility rules are obeyed by the human expert - i.e. the matching and the rankings are not biased by criteria not represented in the knowledge base - then we can indeed create such matching measures, with the help of which the human expertise can be approximated. This shows that the very general approach to matching based on filters provides the necessary flexibility required for diverse matching tasks. 
This is only a starting point for even more sophisticated matching analysis aiming at consensus building among different experts and determination of the most suitable matching measure that is in accordance with the expert knowledge. We also have to take into account that valuations given by human experts will never be complete. This will be addressed in our future research.

\section{References}

1. F. Baader et al., editors. The Description Logic Handbook: Theory, Implementation and Applications. Cambridge University Press, 2003.

2. European distionary of skills and competences. http://www.disco-tools.eu.

3. T. Falk et al. Semantic-Web-Technologien in der Arbeitsplatzvermittlung. Informatik Spektrum, 29(3):201-209, 2006.

4. B. Ganter and R. Wille. Formal concept analysis - mathematical foundations. Springer, 1999.

5. B. C. Grau, I. Horrocks, B. Motik, B. Parsia, P. F. Patel-Schneider, and U. Sattler. OWL 2: The next step for OWL. Journal of Web Semantics, 6(4):309-322, 2008.

6. International standard classification of education. http://www.uis.unesco.org/ Education/Pages/international-standard-classification-of-education.aspx.

7. International standard classification of occupations. http://www.ilo.org/public/ english/bureau/stat/isco/isco08/, 2008.

8. M. Levandowsky and D. Winter. Distance between sets. Nature, 234(5):34-35, 1971.

9. D. Looser, H. Ma, and K.-D. Schewe. Using formal concept analysis for ontology maintenance in human resource recruitment. In F. Ferrarotti and G. Grossmann editors, Ninth Asia-Pacific Conference on Conceptual Modelling (APCCM 2013), volume 143 of CRPIT, pages 61-68. Australian Computer Society, 2013.

10. M. Mochol, H. Wache, and L. J. B. Nixon. Improving the accuracy of job search with semantic techniques. In W. Abramowicz, editor, Business Information Systems, 10th International Conference (BIS 2007), volume 4439 of Lecture Notes in Computer Science, pages 301-313. Springer, 2007.

11. A. L. Paoletti, J. Martinez-Gil, and K.-D. Schewe. Extending knowledge-based profile matching in the human resources domain. In Q. Chen et al., editors, Database and Expert Systems Applications (DEXA 2015) Part II, volume 9262 of LNCS, pages 21-35. Springer, 2015.

12. A. L. Paoletti, J. Martinez-Gil, and K.-D. Schewe. Top-k matching queries for filterbased profile matching in knowledge bases. In H. Ma and S. Hartmann, editors, Database and Expert Systems Applications (DEXA 2016), LNCS. Springer, 2016. to appear.

13. N. Popov and T. Jebelean. Semantic matching for job search engines - a logical approach. Technical Report 13-02, Research Institute for Symbolic Computation, JKU Linz, 2013.

14. G. Rácz, A. Sali, and K.-D. Schewe. Semantic matching strategies for job recruitment: A comparison of new and known approaches. In M. Gyssens and G. R Simari, editors, Foundations of Information and Knowledge Systems (FoIKS 2016), volume 9616 of LNCS, pages 149-168. Springer, 2016. 